\title{
Pathology of Monin-Obukhov similarity in the stable boundary layer
}

\author{
Yinguo Cheng \\ School of Civil and Environmental Engineering, Cornell University, Ithaca, New York, USA
}

Marc B. Parlange

School of Architecture, Civil and Environmental Engineering, Ecole Polytechnique Fédérale de Lausanne, Lausanne, Switzerland

\section{Wilfried Brutsaert}

School of Civil and Environmental Engineering, Cornell University, Ithaca, New York, USA

Received 18 April 2004; revised 29 July 2004; accepted 3 August 2004; published 17 March 2005.

[1] The performance of Monin-Obukhov similarity (MOS) theory has been investigated for four types of events of transient physical processes in the stable nocturnal boundary layer during the Cooperative Atmosphere-Surface Exchange Study-99 (CASES-99). These four types were internal gravity waves, Kelvin-Helmholtz $(\mathrm{K}-\mathrm{H})$ shear instability, a density current, and a low-level jet. It was found that the MOS theory generally holds whenever a new equilibrium is reached or recovered during or after these events but fails in the period of transition or the developing stage of the events with newly generated turbulence. Specifically, MOS was found to hold during the entire process of the nonbreaking internal gravity waves and during the well-developed stages both of the density current and of the low-level jet. It was found not valid during the process of the $\mathrm{K}-\mathrm{H}$ instability and the developing stages of the density current. Characteristic features of these four types of events are also presented and analyzed.

Citation: Cheng, Y., M. B. Parlange, and W. Brutsaert (2005), Pathology of Monin-Obukhov similarity in the stable boundary layer, J. Geophys. Res., 110, D06101, doi:10.1029/2004JD004923.

\section{Introduction}

[2] The accurate determination of turbulent surface fluxes of momentum and heat has always been a problem of central and practical concern in atmospheric science and hydrology. Therefore meteorologists and hydrologists have long relied on similarity theory-based flux profile relationships to estimate fluxes of momentum and heat from their respective profiles of velocity and temperature. The flux profile relationships are also used extensively in numerical models in this regard. The most commonly used flux profile relationships are based on Monin-Obukhov similarity (MOS) theory. It is generally known, however, that computational atmospheric models based on this similarity tend to fail in the stably stratified atmosphere, especially during winter time at higher latitudes. This failure indicates that deficiencies exist in flux parameterization schemes and that the surface fluxes are not always adequately described by the existing MOS theory under such stable conditions. What causes momentum and heat fluxes to depart from the MOS theory is still an open question.

[3] There are several possible reasons for the poor performance of the available flux parameterizations. First, the turbulence is suppressed by the stable stratification itself or

Copyright 2005 by the American Geophysical Union. 0148-0227/05/2004JD004923\$09.00 by the generally weak wind shear under such conditions. For example, in the midlatitudes, particularly under clear skies, the turbulence intensity during nighttime conditions is typically only one tenth of its counterpart in the daytime; as a result this period is often relatively quiescent and accurate measurements are difficult to carry out. Second, the stable boundary layer (SBL) is often characterized by anomalous turbulence events. Intermittent heat, moisture and momentum fluxes are often associated with these anomalies. In contrast to unstable boundary layers with continuous convective turbulence in daytime which have been investigated intensively in recent years [e.g., Brutsaert and Sugita, 1991; Sugita and Brutsaert, 1992; Brutsaert, 1998, 1999], stable boundary layers with intermittent turbulence have received much less attention [Pahlow et al., 2001]; the poor understanding of turbulence intermittency has inhibited the development of reliable parameterizations for the SBL.

[4] A recent field program, the Cooperative AtmosphereSurface Exchange Study-99 (CASES-99), provided unprecedented observational coverage of the nocturnal boundary layer (NBL) over a large vertical and horizontal domain. One of major goals of CASES-99 was to capture the unsteady and transient motions that are so characteristic for stable conditions; in addition, CASES-99 was intended to assess departures from similarity theory under stable conditions and to identify the sources of such departures. Accordingly, the major purpose of the present work is to 
examine the validity of the MOS theory during different types of unsteady and transient behavior under stable conditions and to analyze for each case the specific sources of the departures from similarity theory. The structure of this paper is as follows. The site, measurements and observations of turbulence events are described in section 2; the relevant aspects of MOS theory as well as recent developments are reviewed in section 3 ; section 4 provides results of validation, and major conclusions are given in section 5 .

\section{Site, Measurements, and Turbulence Events}

[5] CASES-99 was the second field campaign of the Cooperative Atmosphere-Surface Exchange Study. It was designed to study events in the nighttime boundary layer, and to investigate the physical processes associated with the evening and morning boundary layer transition regimes. The experimental took place over the period 1-31 October 1999 near Leon, Kansas with a 3 day intercomparison and instrument testing period 27-30 September 1999. The main site $(50 \mathrm{~km}$ east of Wichita, Kansas) covered an area $4.8 \mathrm{~km} \times 3.2 \mathrm{~km}$ with deployment of a large number of instruments to capture stable NBL heterogeneity. These instruments included a heavily instrumented $60 \mathrm{~m}$ tower, numerous $10 \mathrm{~m}$ towers, multiple radars, lidars, scintillometers, tethersondes, rawinsondes and research aircraft.

[6] The CASES-99 data used in this study were collected mainly on the $60 \mathrm{~m}$ tower. Wind and temperature measurements on the $60 \mathrm{~m}$ tower were conducted by sonic anemometers at heights of 1.5, 5, 10, 20, 30, 40, 50, and $55 \mathrm{~m}$. The measurements from the sonic anemometers, which provided three-component wind and virtual temperature data at a sampling rate of $20 \mathrm{~Hz}$, were used in the analysis to calculate the fluxes. These wind measurements, combined with those by prop-vane anemometers, provided wind profiles over the height of the $60 \mathrm{~m}$ tower. Thirty-four thermocouples, thirty-two mounted on the $60 \mathrm{~m}$ tower and the remaining two on two minitowers nearby, were used to measure the air temperature, which was applied in the analysis to provide the temperature gradients and the temperature profiles.

[7] The wind and the temperature measurements used to analyze the spatial variation, were collected by six integrated surface flux facility (ISFF) towers and a high-resolution Doppler lidar (HRDL). The six $10 \mathrm{~m}$ ISFF towers were erected on the main site and surrounded the $60 \mathrm{~m}$ main tower. On each ISFF tower, a sonic anemometer was mounted at $5 \mathrm{~m}$ with a sampling rate of $20 \mathrm{~Hz}$, a prop-vane anemometer for wind measurement at $10 \mathrm{~m}$ with a sampling rate of $1 \mathrm{~Hz}$. The High Resolution Doppler Lidar (HRDL), was a scanning type, coherent Doppler lidar system designed for atmospheric boundary layer research [Newsom and Banta, 2002]; it was deployed by the Environmental Technology Laboratory (ETL) of the National Oceanic and Atmospheric Administration (NOAA) at a site approximately $1.45 \mathrm{~km}$ south of the $60 \mathrm{~m}$ tower. This HRDL could map out the wind field over a 2-D section of the atmosphere by scanning in either azimuth or elevation.

[8] The measurements from mini sodars and wind profilers were used to provide vertical information above the $60 \mathrm{~m}$ tower. Additionally, microbarographs sampling to $1 \mathrm{~Pa}$ at $2 \mathrm{~Hz}$ were mounted at $1 \mathrm{~m}, 30 \mathrm{~m}$ and $50 \mathrm{~m}$, with the intent of providing a record of coherent pressure disturbances in the vertical direction. More detailed information can be found in a comprehensive review of CASES-99 by Poulos et al. [2002].

[9] Throughout the field phase of CASES-99, the realtime instrumentation captured significant turbulence events and provided a time history of these events to allow an evaluation of their contributions to intermittent heat and momentum fluxes. In the present study, we focus on an occurrence of each of the following four atmospheric phenomena, namely an event of internal gravity waves, a Kelvin-Helmholtz (K-H) shear instability, a density current front and a low-level jet. The total number of such events were observed [Poulos et al., 2002] to be: At least one K-H shear instability event, 10 density current passages, 13 lowlevel jets, and 7 other events including large amplitude wave activities.

\section{Brief Review of MOS Theory}

[10] Flux profile relationships relate the turbulent flux of momentum and heat to their respective profiles of mean velocity and temperature. The most commonly used flux profile relationships are based on the MOS theory, which predicts that the dimensionless gradient of the mean wind speed $\bar{u}$ and of the mean potential temperature $\bar{\theta}$ are universal functions of atmospheric stability

$$
\begin{aligned}
& \frac{\kappa\left(z-d_{0}\right)}{u_{*}} \frac{d \bar{u}}{d z}=\phi_{m}(\zeta) \\
& \frac{\kappa\left(z-d_{0}\right)}{T_{*}} \frac{d \bar{\theta}}{d z}=\phi_{h}(\zeta)
\end{aligned}
$$

where the subscripts $m$ and $h$ refer to momentum and sensible heat respectively, an overbar denotes mean quantity, $\kappa$ is von Kármán's constant, $z$ the height above the ground, $d_{0}$ the displacement height; the friction velocity $u *$ is defined as follows:

$$
u_{*}=\sqrt{\frac{\tau_{0}}{\rho}}
$$

in which $\rho$ is the air density and $\tau_{0}$ is the surface shear stress. The latter is related to the turbulence covariance by

$$
\tau_{0}=-\rho \overline{u^{\prime} w^{\prime}}
$$

where $u^{\prime}$ and $w^{\prime}$ are the longitudinal (i.e., streamwise) and vertical velocity fluctuations, respectively.

[11] The stability variable is defined as

$$
\zeta=\frac{z-d_{0}}{L}
$$

where $L$ is the Obukhov length scale defined as

$$
L=\frac{-\rho u_{*}^{3}}{k g \frac{H_{v}}{T_{0} c_{p}}}
$$


[12] The symbol $T_{0}$ denotes the averaged temperature near the ground, $c_{p}$ the specific heat at constant pressure, $g$ the acceleration of gravity, and $H_{v}$ the turbulent surface flux of virtual sensible heat, which is given by

$$
H_{v}=-\rho c_{p} \overline{w^{\prime} \theta_{v}^{\prime}}
$$

where $\theta_{v}^{\prime}$ is virtual temperature fluctuation.

[13] For computational purposes it is often convenient to express the stability functions for wind speed and temperature in integral form

$$
\Psi(\zeta)=\int_{0}^{\zeta} \frac{[1-\phi(x)]}{x} d x
$$

in which for brevity of notation, the subscripts $m$ and $h$ are omitted.

[14] The most commonly used gradient stability functions under stable conditions have the following linear forms

$$
\begin{gathered}
\phi_{m}=\left(1+\beta_{m} \zeta\right) \\
\phi_{h}=a_{h}^{-1}\left(1+\beta_{h} \zeta\right)
\end{gathered}
$$

for values of $\zeta$ smaller than an upper limit of $\zeta_{c}$ of the order of one; the symbol $a_{h}$ denotes the ratio of the von Kármán constants for sensible heat and momentum, and it is usually treated as unity. For $\zeta>\zeta_{c}$, both $\phi$ functions appear to remain approximately constant [Webb, 1970; Hicks, 1976; Kondo et al., 1978]. Because exact forms of these functions and the values of the parameters $\beta_{m}$ and $\beta_{h}$ are not predicted by theory, Cheng and Brutsaert [2005] re-examined these functions using wind and temperature measured during the CASES-99 and found these parameters to be $\beta_{m}=5.8$ and $\beta_{h}=5.4$ with $\zeta_{c}=0.8$.

[15] To cover a wider stability range, a single general formulation was proposed which recovers the linear formulation and the constant at the two extremes, i.e., small and large values of $\zeta$, with a smooth transitional region

$$
\phi(\zeta)=1+a \frac{\zeta+\zeta^{b}\left(1+\zeta^{b}\right)^{\frac{1-b}{b}}}{\zeta+\left(1+\zeta^{b}\right)^{\frac{1}{b}}}
$$

where $a$ and $b$ are constants and are found to be $a=6.1$ and $b=2.5$ for wind speed and $a=5.3$ and $b=1.1$ for temperature.

[16] The linear and the general formulations in section 3 are used to check the validity of the MOS theory during four events mentioned in section 2 . The general characteristics of these four events will be given but sources of these phenomena are out of the scope of this work and not described herewith. The universal time coordinated (UTC) was used in the analysis, which is 6 (5) hours ahead of the Central standard (daylight saving) time.

\section{Analysis and Results}

\subsection{Averaging Scheme and Averaging Time}

[17] In the lower atmosphere the turbulent fluxes are commonly estimated as covariances by means of Reynolds averaging over periods of the order of $30 \mathrm{~min}$ to 1 hour.
However, as also discussed elsewhere [Cheng and Brutsaert, 2005], because of the highly transient nature of the four events considered here, the commonly used Reynolds averaging method is likely to suffer from a great deal of uncertainty in the flux computations. To reduce this uncertainty, it was decided to use running averaging schemes with adaptive averaging periods instead.

[18] In the study of turbulence, it is important not to confuse it with wave motion. Therefore in the analysis of the events involving nonbreaking gravity waves and K-H shear instability a triple-decomposition scheme was used with a linear filtering technique in order to capture beside the turbulence also wave motions with characteristic timescales from a few seconds to several minutes. The tripledecomposition is a generally accepted and time-tested procedure to separate mean, wave and turbulent motions instantaneously [e.g., Benilov and Filyushkin, 1970; Lumley and Terray, 1983; Jiang et al., 1990]; in the case of instantaneous measurements of pressure, wind velocity and temperature, the decomposition can be formulated as follows

$$
\begin{gathered}
p=\bar{p}+\widetilde{p}+p^{\prime} \\
u_{i}=\overline{u_{i}}+\widetilde{u_{i}}+u_{i}^{\prime} \\
\theta=\bar{\theta}+\widetilde{\theta}+\theta^{\prime}
\end{gathered}
$$

in which the overbar, the tilde and the prime represent the mean, the wave-induced component and the turbulent fluctuation, respectively, and the subscripts represent velocity components in the $x, y$, and $z$, respectively. There are several ways of implementing this basic idea of tripledecomposition [e.g., Hussain and Reynolds, 1972; Hussain, 1983; Finnigan et al., 1984]. However, for the specific purpose of the present study it was decided to carry out the triple-decomposition using band-pass filtering, which had already been widely used to separate the mean variations from turbulence fluctuations [Lenschow et al., 1988; Smedman et al., 1993]. Because each variable has three components, this involved two separate filtering processes each with a box filter function [e.g., Pope, 2000, p. 579]. The first one used a high-pass filter with a smaller window size to extract the turbulent component from the instantaneous measurements. The second one used a low-pass filter with a larger window size to get the mean quantities. In other words, the triple decomposition technique was applied with a large window to obtain the mean quantities and with a small window to obtain the turbulence; the wave component could then be obtained by subtraction of the mean and turbulent components from the instantaneous measurements. The advantage of this band-pass filtering technique is that it allows the decomposition of a timedependent variable into its three components at any measuring instant. The adopted averaging period must depend on the event duration and on the wave period. The determination of averaging time span for the mean was based on the following considerations:

[19] 1. It should not exceed the entire duration of the event. 


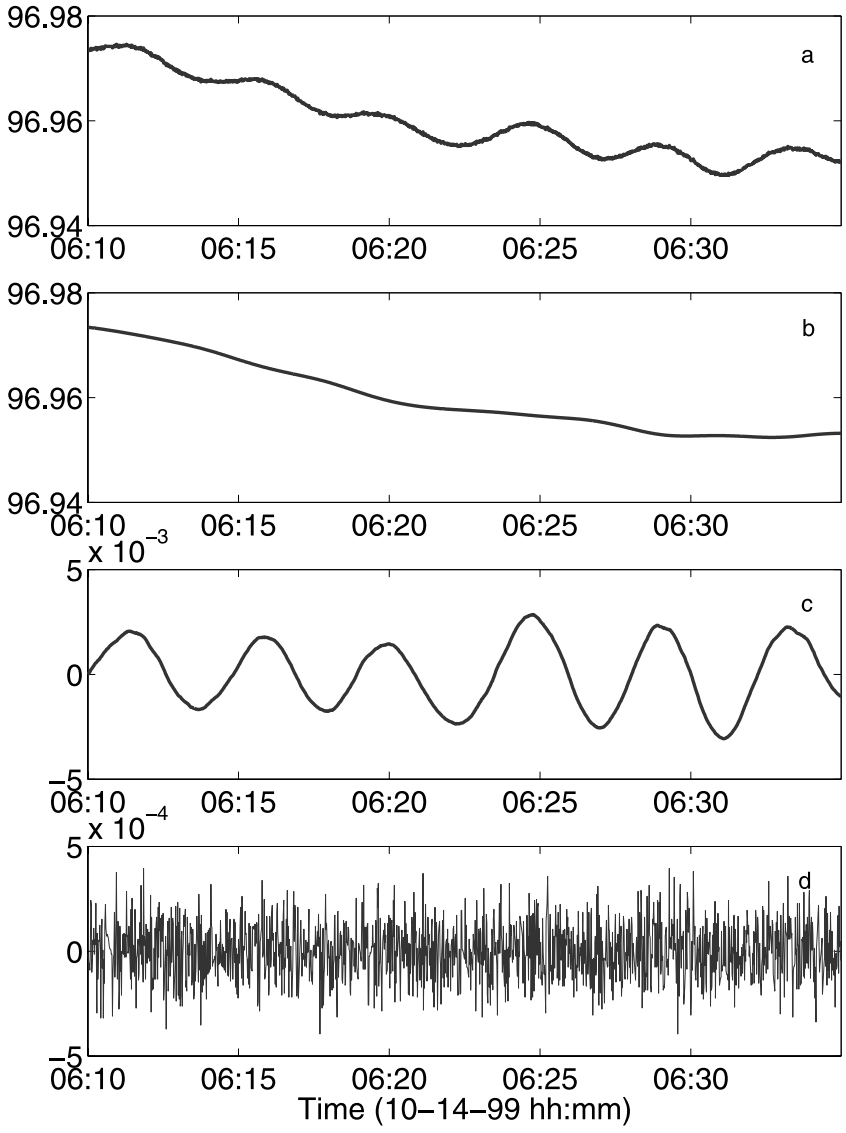

Figure 1. Triple-decomposition of the pressure at $1.5 \mathrm{~m}$ during the nonbreaking wave event between 0610 and 0635 UTC on 14 October 1999. (a) The instantaneous pressure measurement $p$. (b) The mean value of the pressure $\bar{p}$. (c) The wave component of the pressure $\tilde{p}$. (d) Turbulence fluctuation of the pressure $p^{\prime}$ after decomposition. See color version of this figure in the HTML.

[20] 2. It should cover at least a complete wave cycle.

[21] 3. It cannot be so long as to include transient effects because wave is evolving itself. Since often no clear gap exists between wave and turbulence, the cutoff frequency, i.e., the inverse or the reciprocal of the smaller window size, was determined by trial and error in order to extract the clear waveform and to make sure that the mean of the turbulent quantities was as close to zero as possible.

[22] For the density current and for the low-level jet events studied here, the instantaneous quantities could be simply separated into the mean and turbulence since no wave motion was involved. For these two types of events, a 5-min running averaging was used, as this appeared to be adequate to capture the transient effects. Turbulence was computed after subtracting the mean quantities.

\subsection{Nonbreaking Internal Gravity Waves}

[23] Internal gravity wave episodes are always present in the stable boundary layer over periods of seconds to a few minutes [Gossard and Hooke, 1975, p. 388]. Such internal waves can have the appearance of nonbreaking wave packets, breaking waves, braids, or cat's eyes. In this section, the focus is on nonbreaking waves. A typical wave event occurred between 0610 and 0640 UTC on 14 October 1999. On the basis of a linear filtering technique, the measurements were triply decomposed as indicated above in (12), (13) and (14).

[24] This triple decomposition was achieved by using the two filtering processes, each with a box filter function, as explained in section 4.1. The smaller and larger window sizes were obtained by trial and error as 0.35 and 5 min. This means that the wave information was assumed to be contained between the frequencies of 0.0476 and $0.0033 \mathrm{~Hz}$.

[25] Figure 1a shows the instantaneous pressure measurement at the height of $1.5 \mathrm{~m}$. After filtering, the mean pressure, the wave and the turbulence components of the pressure are shown in Figures $1 \mathrm{~b}, 1 \mathrm{c}$, and 1d, respectively. The dominant frequency of the waves is $0.0038 \mathrm{~Hz}$ and the amplitude is of the order of $5 \times 10^{-3} \mathrm{kPa}$. A nearly stationary turbulence can be seen in Figure 1d. However, the displayed turbulence probably still contains a great deal of measurement noise due to the accuracy of microbarograph.

[26] Wave features were also obtained from wind and potential temperature measurements by using the same triple-decomposition scheme. In Figures $2 \mathrm{a}$ and $2 \mathrm{~b}$, the resulting mean horizontal wind speed and the wave oscillations are presented. The corresponding mean potential temperature and the wave components are shown in Figures $2 \mathrm{c}$ and $2 \mathrm{~d}$. The wave oscillations of the potential temperature are nearly in phase with waves of the wind speed. However, the waves in the wind speed and in the potential temperature are not as smooth and regular as in the pressure. This could be due to the presence of turbulence scales in pressure which are different from those in wind and in potential temperature. This scale difference, in turn, could conceivably be caused by the nonlinear interactions between the turbulence and wave-induced changes in wind and in potential temperature. This topic is currently being investigated.

[27] A comparison between the experimental and the theoretical $\phi$ values at a height of $3.25 \mathrm{~m}$ is presented in Figure 3. Once the running averaging scheme has been selected, computations of the similarity function can be carried out at any time resolution ranging from the measuring interval to the averaging period. The similarity functions were computed every minute to follow their behavior during the wave cycles. (Note that this 1-min time interval was not an averaging period, but rather the frequency with which the instantaneous values were calculated over the selected windows). The top panel shows a comparison of the calculated $\phi_{m}$ values for the wind speed, and the bottom one shows a comparison of the calculated $\phi_{h}$ for potential temperature. The experimental $\phi$ data points (circles) were calculated by (1) and (2); the theoretical predictions were obtained from the linear formulations (squares), namely (9) and (10), and from the general function (11) (stars). It can be seen that the theoretical predictions match the experimental results reasonably well both for the wind speed and the temperature except at the left end. The theoretical predictions with the general function (11) are closer to the experimental data points than those with the linear formulations. It would seem that the discrepancy at the left end is directly related to random flux errors and 

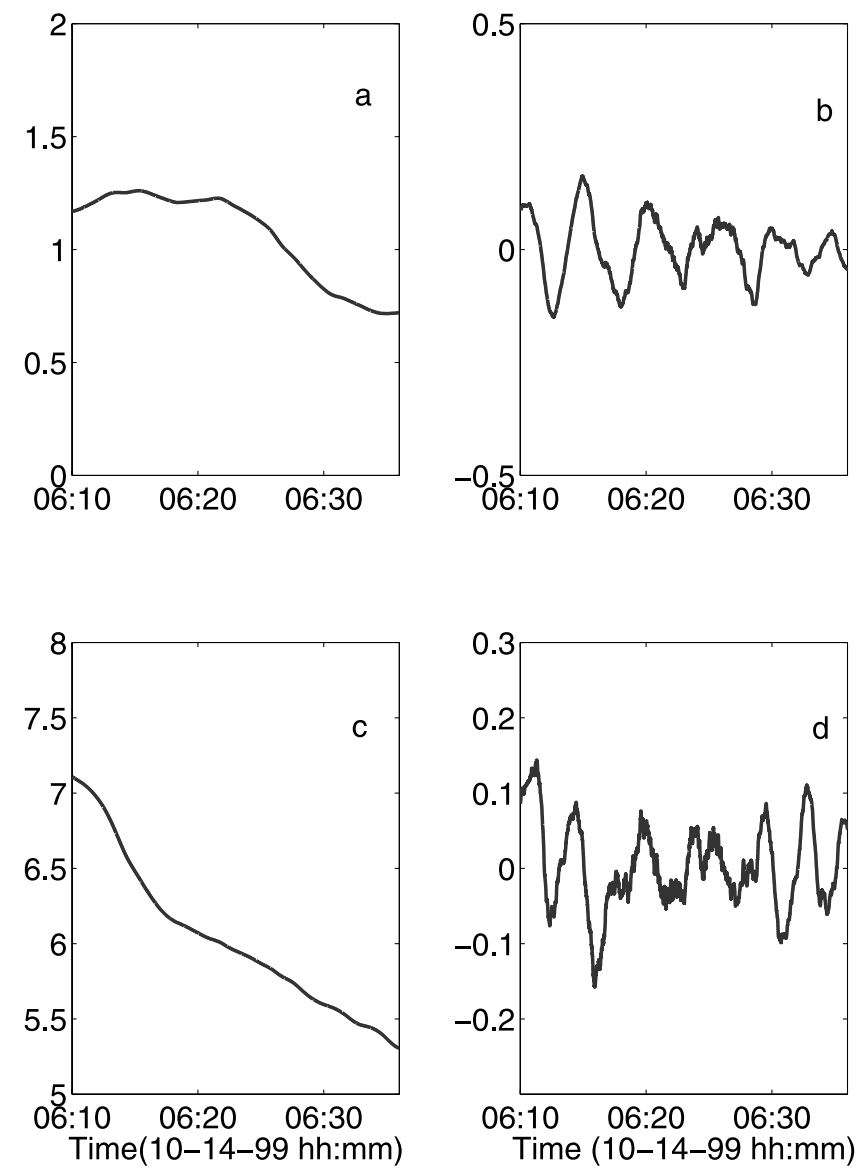

Figure 2. The mean and the wave component of the wind speed and of the potential temperature at $1.5 \mathrm{~m}$ after triple decomposition during the nonbreaking wave event between 0610 and 0635 UTC on 14 October 1999. (a) The mean wind speed $\bar{u}$. (b) The wave component of the wind speed $\tilde{u}$. (c) The mean potential temperature $\bar{\theta}$. (d) The wave component of the potential temperature $\tilde{\theta}$. See color version of this figure in the HTML.

instrument errors; indeed, as seen in (1) and (2), larger values of the $\phi$ functions, are the result of smaller flux values, which are more difficult to measure.

[28] On the basis of these comparisons, it can be concluded that the MOS theory is valid during this nonbreaking wave event. This could be explained as follows. First, the passage of the nonbreaking waves does not dramatically change the ambient mean wind and the potential temperature gradient. Second, the interaction between the waves and the turbulence is small because of the large spectral gap between the waves and the turbulence, and the wave passage is unlikely to contaminate the ambient mean and the underground turbulence fields. This suggests that it is safe to apply the MOS theory to nonbreaking wave events provided no other mechanism of turbulence generation is involved.

\subsection{Kelvin-Helmholtz Shear Instability}

[29] In contrast to internal gravity waves, waves excited by the Kelvin-Helmholtz shear instability mechanism are breaking. In this case, a stratified shear flow excites certain wave modes which grow until they become unstable and finally break. By this breaking process, the waves dissipate their energy into high frequency motions. New turbulence is thus generated, and it is often manifested by a sudden outbreak of turbulence.

[30] A typical event of the K-H shear instability was captured between 0515 and 0545 UTC on 6 October 1999. Some general features of this event were already documented in earlier studies by Blumen et al. [2001] and Newsom and Banta [2002]. For the present study the relevant information can be derived from the pressure, the wind and the potential temperature measurements on the $60 \mathrm{~m}$ tower; these are shown in Figures $4 \mathrm{a}, 4 \mathrm{~b}$, and $4 \mathrm{c}$, respectively. Figure $4 \mathrm{~d}$ shows the turbulence fluctuations of the potential temperature. Separation of the wave and the turbulence from the instantaneous measurements was carried out by the same triple decomposition scheme as before with small and large windows of $0.2 \mathrm{~min}$ and $4 \mathrm{~min}$, respectively. From the intensity of the turbulence in Figure $4 \mathrm{~d}$, it can be inferred that the waves started breaking at around 0515 UTC and the breaking subsided at about 0545 UTC. Interestingly, newly generated turbulence can be seen under each crest of the breaking waves at the beginning stage. Turbulence intensity increases abruptly each time when a wave collapses. The root mean square of the potential temperature turbulence is, for example, $0.26^{\circ} \mathrm{C}$ from 0505 to 0515 UTC prior to the wave breaking; it rises to $0.52^{\circ} \mathrm{C}$ from 0525 to 0545 UTC during the event, and it subsides again down to $0.23^{\circ} \mathrm{C}$ from 0550 to 0600 UTC
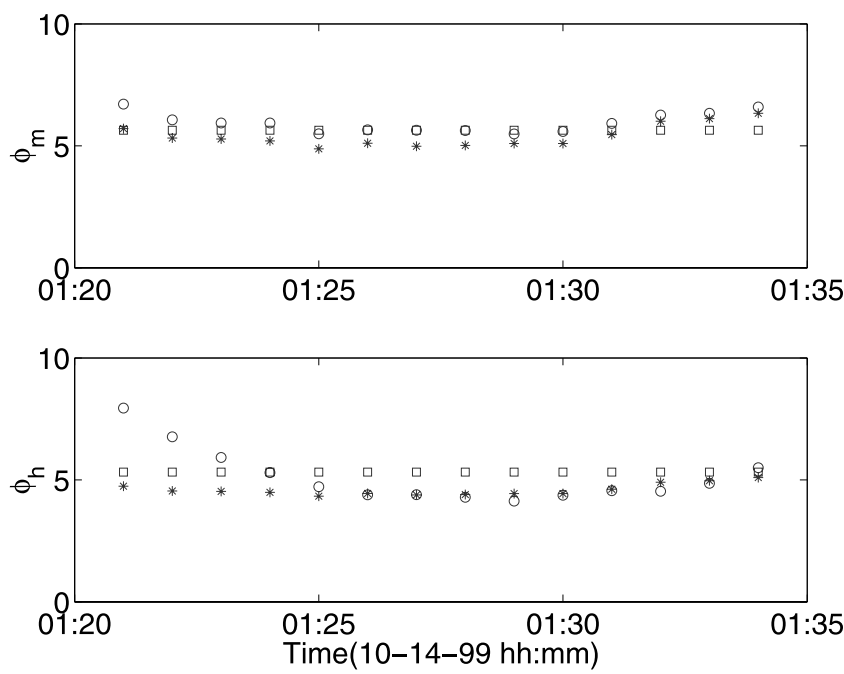

Figure 3. Comparisons of the experimental $\phi$ data points (circles) at a height of $3.25 \mathrm{~m}$ with the theoretical predictions (squares, by the linear formulations; stars, by the general stability function) during the nonbreaking wave event. The top panel is a comparison of the experimental $\phi_{m}$ values calculated by (1) with the theoretical predictions by (9) with $\beta_{m}=5.8$ and by (11) with $a=6.1$ and $b=2.5$ for the wind speed; the lower panel is a comparison of the experimental $\phi_{h}$ values calculated by (2) with the theoretical predictions by (10) with $\beta_{h}=5.4$ and by (11) with $a=5.3$ and $b=1.1$ for the temperature. See color version of this figure in the HTML. 

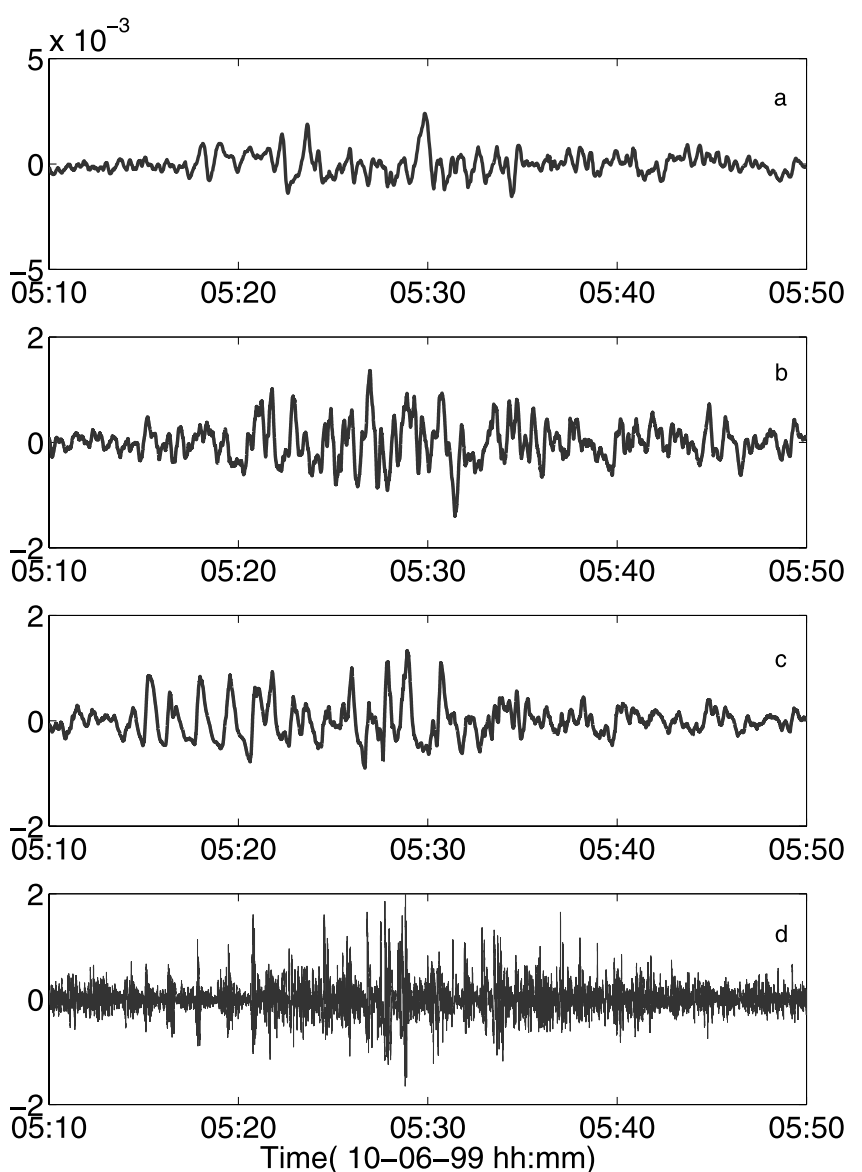

Figure 4. Triple decomposition of the pressure, the wind speed, and the potential temperature during the K-H shear instability event between 0510 and 0550 UTC on 6 October 1999. (a-c) The wave component of the pressure, of the wind speed, and of the potential temperature, respectively. (d) The turbulence fluctuations of the potential temperature after decomposition. See color version of this figure in the HTML.

after the event. Its intensity increases by $100 \%$ due to the wave breaking and decays to its original level after the breaking.

[31] Figure 5 shows a comparison of the calculated $\phi$ data points (circles) at a height of $3.25 \mathrm{~m}$ with the theoretical MOS predictions by the linear formulations (9) and (10) (squares) and by the general stability function (11) during this event. The top frame shows the calculated $\phi_{m}$ values and the lower one the calculated $\phi_{h}$ values. It can be seen that the predictions match the experimental data quite well before and after the wave breaking. However, the MOS theory predicts larger $\phi$ values than the experimental results during the breaking. For example, the linear formulation and the general stability function overestimate the $\phi_{h}$ values by $230 \%$ and by $156 \%$, respectively over the breaking period. Again, the general function (11) predicts $\phi$ values closer to the experimental data than the linear formulations do. The same trend can be noticed in both comparisons.

[32] As can be seen in (1) and (2), an overprediction of $\phi_{m}$ and $\phi_{h}$ should normally lead to an underprediction of the surface momentum flux and of the heat flux during the breaking period. In order to further strengthen this observation, the friction velocity $u_{*}$ and the scaling temperature $T *$ were calculated from the flux profile relationships in the following integral form

$$
\begin{gathered}
\bar{u}=\frac{u_{*}}{k}\left[\ln \left(\frac{z}{z_{0}}\right)-\Psi_{m}\right] \\
\Delta \theta=\frac{T_{*}}{k}\left[\ln \left(\frac{z}{z_{\text {ref }}}\right)-\Delta \Psi_{h}(\zeta)\right]
\end{gathered}
$$

where $\Delta \Psi_{h}$ is the difference of $\Psi_{h}$ and $\Delta \zeta$ the difference of $\zeta$ between the level of interest $z$ and a reference level $z_{\text {ref }}$.

[33] Figure 6 shows a comparison between the experimental data of $u *$ and of $T *$ and the corresponding solutions obtained from (15) and (16). It can be seen that the calculated values for $u_{*}$ and $T_{*}$ are indeed lower than the experimental values. On average, the MOS predictions underestimate $u_{*}$ by roughly $40 \%$ and $T_{*}$ by about $52 \%$ during the wave breaking episode.

\subsection{Density Current}

[34] A density current, sometimes also called a gravity current or buoyancy current, can occur in coastal regions, inland sites and hilly terrain in different ways in the atmosphere [Lapworth, 2002]; it can occur at relatively large scales, ranging from synoptic fronts down to individual thunderstorms, with depths of $\mathrm{O}(1000 \mathrm{~m})$ and advancing speeds of $\mathrm{O}\left(10 \mathrm{~m} \mathrm{~s}^{-1}\right)$ [Simpson, 1997, p. 1], or at considerably smaller scales, such as a shallow drainage flow close to ground level of $\mathrm{O}(10 \mathrm{~m})$ with much smaller propagation speeds [Blumen et al., 1999; Mahrt et al., 2002]. The latter currents, which are of interest here, are
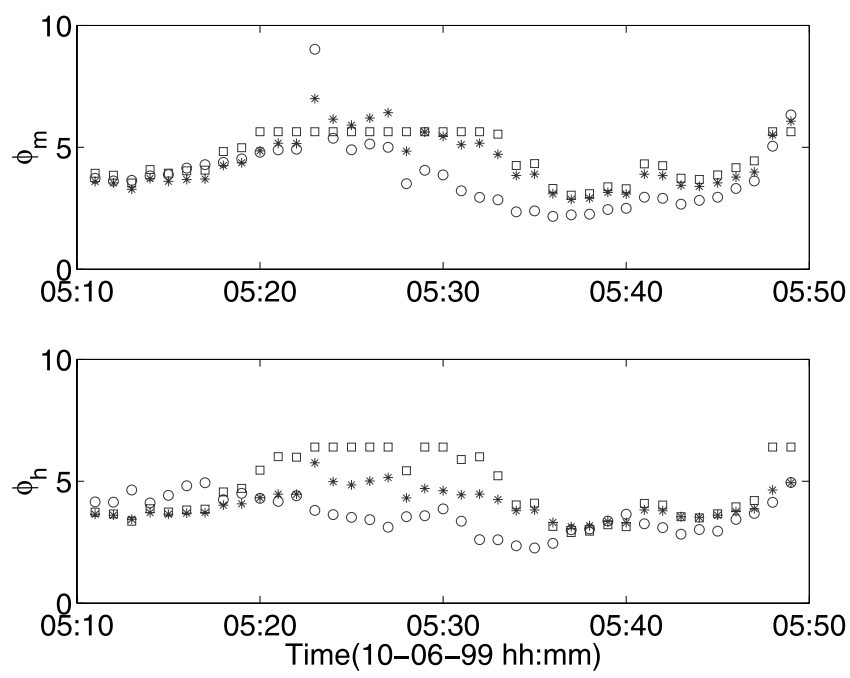

Figure 5. Comparisons of the experimental $\phi$ data points (circles) at a height of $3.25 \mathrm{~m}$ with the theoretical predictions by the linear formulations (squares) and by the general stability function (stars) during the K-H shear instability event. The structure of the figure is the same as that of Figure 3. See color version of this figure in the HTML. 

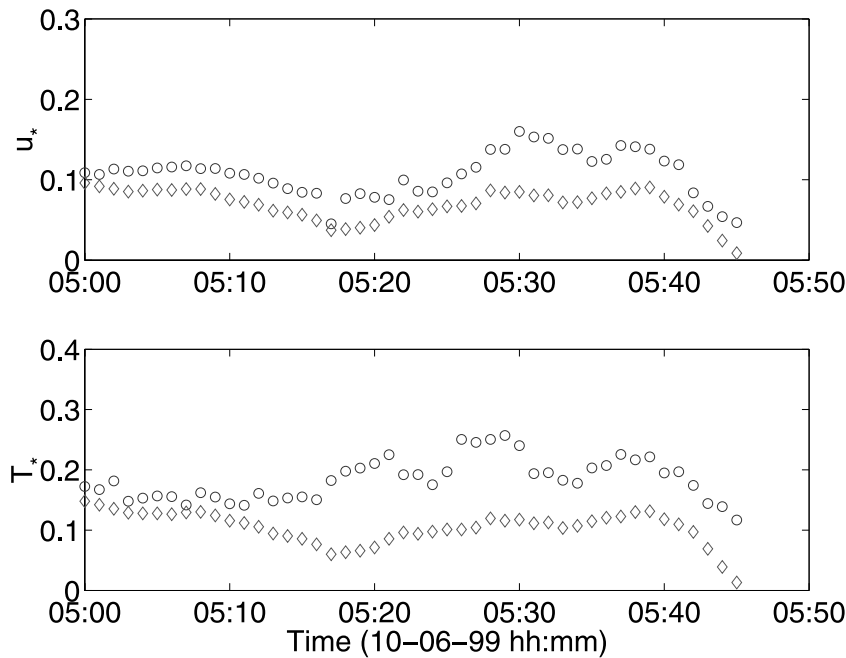

Figure 6. A comparison between the experimental data (circles) and the values obtained from the flux-profile relations (15) and (16) (diamonds) during the K-H shear instability event for the friction velocity $u_{*}$ (top frame) and for the scaling temperature $T_{*}$ (bottom frame). See color version of this figure in the HTML.

primarily generated by density differences of only a few percent.

[35] A shallow density current was recorded by the CASES-99 instruments on 18 October 1999. The structure of the density current can be seen in the time-height cross section of the potential temperature in Figure 7. Several features can be noticed. The nose of the density current or its earliest arrival, about $1 \mathrm{~m}$ above the ground surface, is clearly visible in the contour lines $3.5,4$ and $4.5^{\circ} \mathrm{C}$. The head, which is the top of the leading edge evolving between 0145 and about $0200 \mathrm{UTC}$, is higher than the rest of the density current. A solitary wave can be observed above the head in contour lines $6,6.5,7$ and $7.5^{\circ} \mathrm{C}$ and is propagating

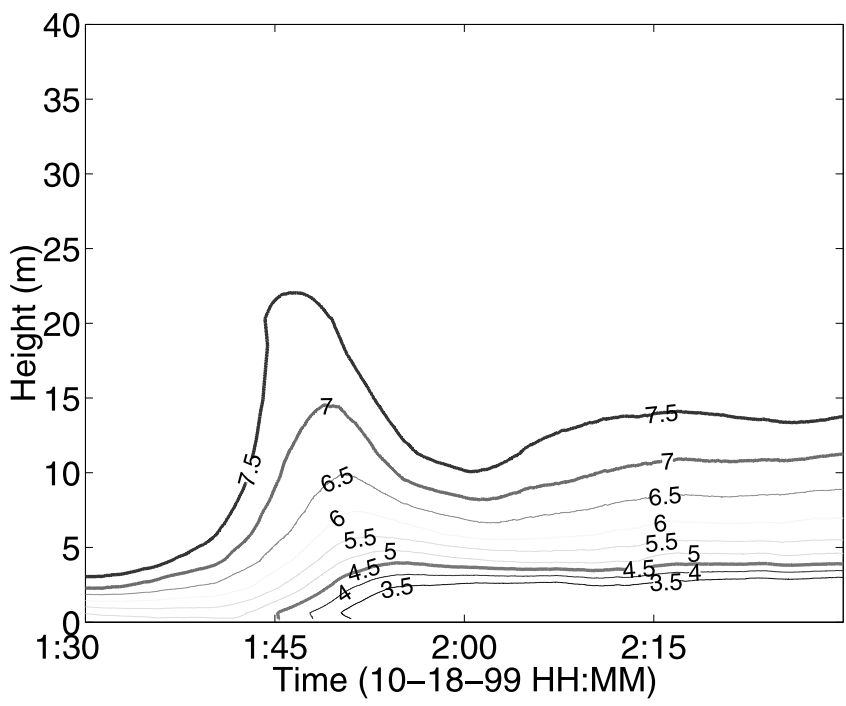

Figure 7. A time-height cross section of the potential temperature during the passage of the density current between 0130 and 0230 UTC on 18 October 1999. See color version of this figure in the HTML. horizontally and vertically. An internal hydraulic jump can be seen in the contour lines above the density current (e.g., $5.5^{\circ} \mathrm{C}$ and $6^{\circ} \mathrm{C}$ ) and is moving with the density current.

[36] Figure 8 shows the time history of the wind speed and of the potential temperature at 4 levels, namely 5, 15, 25 and $35 \mathrm{~m}$, before and during the passage of the density current from 0130 to 0220 UTC. Starting at 0145 UTC, the wind speed rapidly increases by 2 to $3 \mathrm{~m} \mathrm{~s}^{-1}$ in conjunction with a temperature drop of about $2^{\circ} \mathrm{C}$ except at the lowest level. The temperature drop was followed by an increase of about 2 to $3^{\circ} \mathrm{C}$ when the head of the density current was passing. The increase in velocity was obviously related to the advancing speed of the density current which was faster than the speed of the prior ambient flow. The cold air, carried by the current, led to a temperature drop at the lower levels (namely, 5 and $15 \mathrm{~m}$ ). The up and the down in the potential temperature at the higher levels (namely, 25 and $35 \mathrm{~m}$ ) can be attributed to the solitary wave above the head. The recovery in the potential temperature at higher levels above $15 \mathrm{~m}$ was due to a descending motion in the wake behind the solitary wave of a large amplitude. After a transitional period of about $12 \mathrm{~min}$ associated with the head, the wind speed and the potential temperature gradually stabilized as the density current became well developed and reached a steady state. By analysis of measurements on the ISFF and main towers and of the minisodar observations in an earlier study, Sun et al. [2002] concluded that the propagation speed and the height of this current were $2.3 \mathrm{~m} \mathrm{~s}^{-1}$ and $120 \mathrm{~m}$.

[37] Figure 9 shows profiles of the wind speed and of the potential temperature at 0135 (circles), 0215 (pluses) and 0220 (stars) UTC before and after the head passage. The increase in the wind speed and the decrease in the potential temperature are most obvious close to the ground. For instance, after the head passage, the difference between
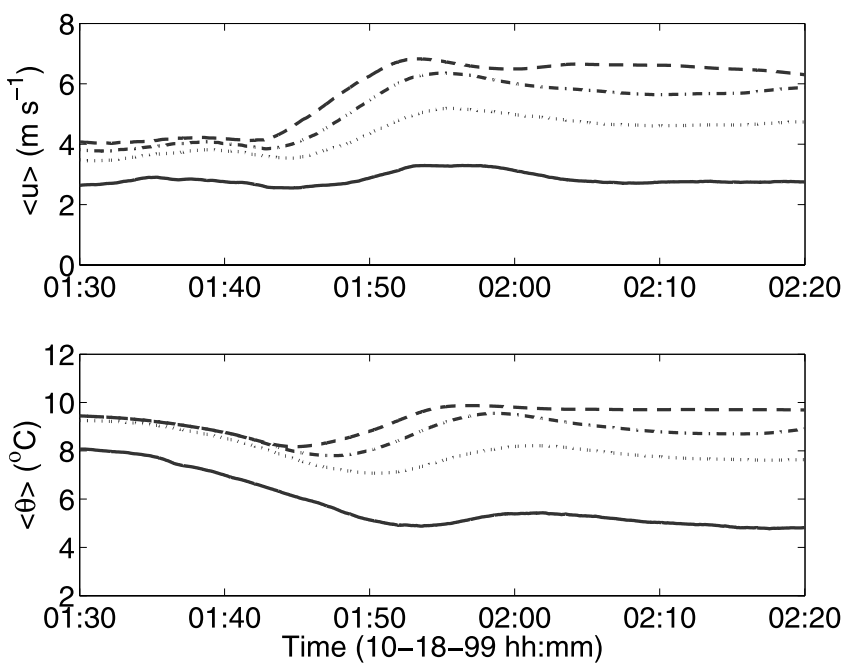

Figure 8. Time history of the wind speed and of the potential temperature during the passage of the density current between 0130 and 0220 UTC on 18 October 1999. The upper frame is for the wind speed, and the lower one for the potential temperature. The measurements were made at $5 \mathrm{~m}$ (solid line); $15 \mathrm{~m}$ (dot line); $25 \mathrm{~m}$ (dash-dot line), and $35 \mathrm{~m}$ (dash line). The notation \langle\rangle is equivalent with overbar in the text. See color version of this figure in the HTML. 

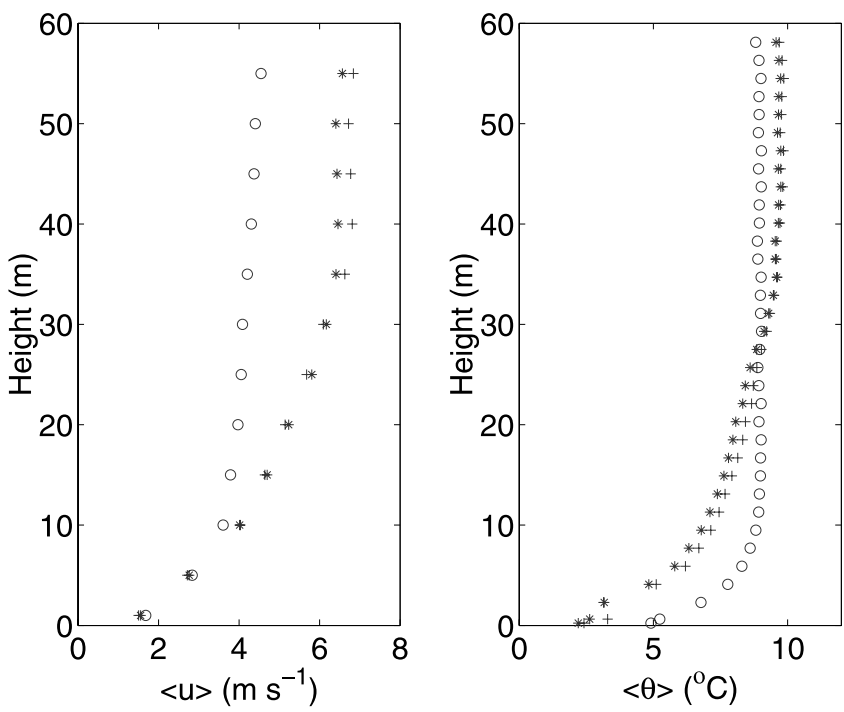

Figure 9. (left) Profiles of the wind speed and (right) the potential temperature before and after the arrival of the density current at 0135 (circles), 0215 (pluses), and 0220 (stars) UTC. Notation in brackets is equivalent with overbar in the text. See color version of this figure in the HTML.

the wind at $20 \mathrm{~m}$ and at the surface (as a measure of wind shear) increased by $91 \%$; in a similar way this temperature difference (as a measure of the temperature gradient) increased by $56 \%$. The collapse of the two profiles of the wind and of the potential temperature at 0215 (pluses) and 0220 (stars) UTC indicates that the density current was well developed and had reached a steady state.

[38] Figure 10 shows comparisons of the experimental $\phi$ values (circles) at a height of $3.25 \mathrm{~m}$ with the theoretical values by the linear formulations (9) and (10) (squares) and by the general function (11) (stars). The upper panel shows the $\phi_{m}$ values and the lower one the $\phi_{h}$ values. The $\phi_{m}$ values seem to agree overall, although a difference can be seen between 0204 and 0211 UTC. However more pronounced discrepancies occurred in the $\phi_{h}$ values between 0145 and 0201 UTC during the head passage and between 0204 and 0211 UTC. During the head passage the theoretical predictions of the $\phi_{h}$ values are smaller than the experimental values. This could be due to the warmer air carried by a descending motion behind the solitary wave, which resulted in larger temperature gradients. Apparently the wind speed gradient remained less affected by this. The differences in the $\phi_{m}$ and the $\phi_{h}$ values between 0204 and 0211 UTC are probably due to the small shear instability, already noticed by Sun et al. [2002], which occurred on the interface between the density current and warmer air above it. However, here the theoretical MOS values are larger than the experimental values, this is the same behavior as already noticed in the case of the K-H instability (see Figure 5). Again, the general function (11) performs better than the linear formulations (9) and (10).

[39] The departure of the surface heat flux and, to a lesser extent, of the surface momentum flux from the MOS theory during the head passage can be attributed to the intermittent flow structure associated with the head of the density current. Immediately, behind the head the density current appears to be fully developed; however, because there is a small shear instability on the "back" of this current, MOS is still not applicable. It is only after this instability has dissipated, i.e., after 0211 UTC, that the surface fluxes can be predicted by the MOS theory. At this stage the turbulence has become continuous (i.e., nonintermittent) and stationary. A new equilibrium is thus established in the mean flow and in the turbulence after disruptions by the front head have fully dissipated.

\subsection{Low-level Jet}

[40] The atmospheric LLJ refers to any low-level speed maximum in the vertical profile of wind speed, and it can be produced by a number of mechanisms [e.g., Banta et al., 2002]. The LLJ has been well documented in the SBL and intensively studied [Smedman et al., 1993, 1995; Zhong et al., 1996; Poulos et al., 2002; Banta et al., 2002]. The frequency of occurrence, the spatial distribution and the evolution of the LLJs, which occurred during CASES-99, were discussed by Banta et al. [2002]. The wind maximum was generally at or below $100 \mathrm{~m}$ above ground level. The LLJ, used as an example in the present study, occurred on 11 October 1999. Its general structure can be inferred from the recorded sounding and profiler data [Poulos et al., 2002, Figure 19]. The entire jet development took place below $600 \mathrm{~m}$ and the center of the jet with maximum velocity was located at about $150 \mathrm{~m}$ above ground level. This LLJ persisted for a long time through the night and became fully developed by 0700 UTC with a maximum wind speed of $18 \mathrm{~m} \mathrm{~s}^{-1}$. The jet maximum remained constant through 1200 UTC, and started to diminish after that. The establishment of this well developed jet can be seen from the temporal variations of the momentum and the heat flux. In Figure 11, the downward momentum (top panel) and the downward heat (bottom panel) flux increased until 0700 UTC. These fluxes stayed more or less constant after the jet maximum had been reached at 0700 UTC. Evidently,
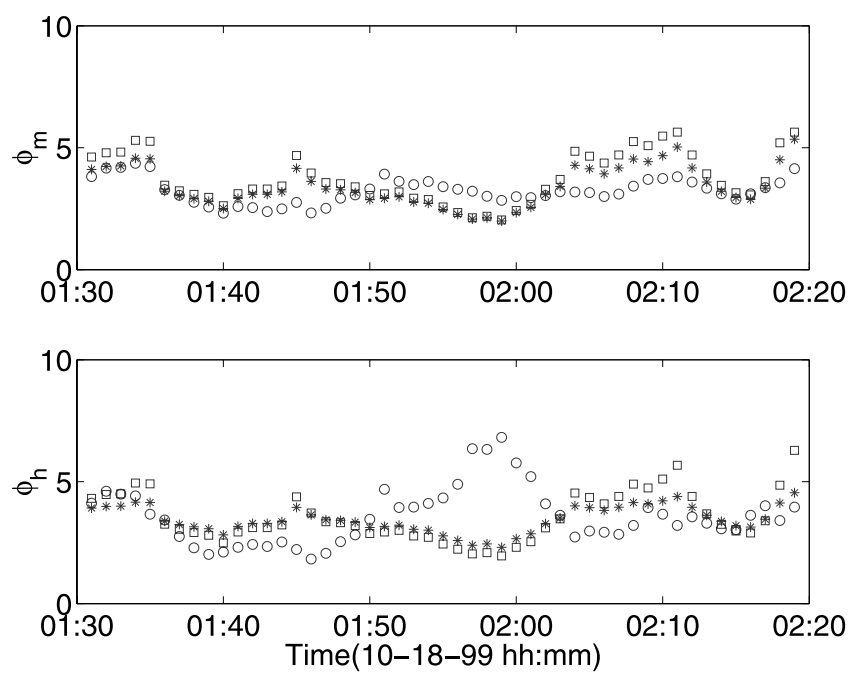

Figure 10. Comparisons of the experimental $\phi$ data points (circles) at $3.25 \mathrm{~m}$ above the ground with the theoretical predictions by the linear formulations (squares) and by the general stability function (stars) during the density current event. The structure of the figure is the same as that of Figure 3. See color version of this figure in the HTML. 

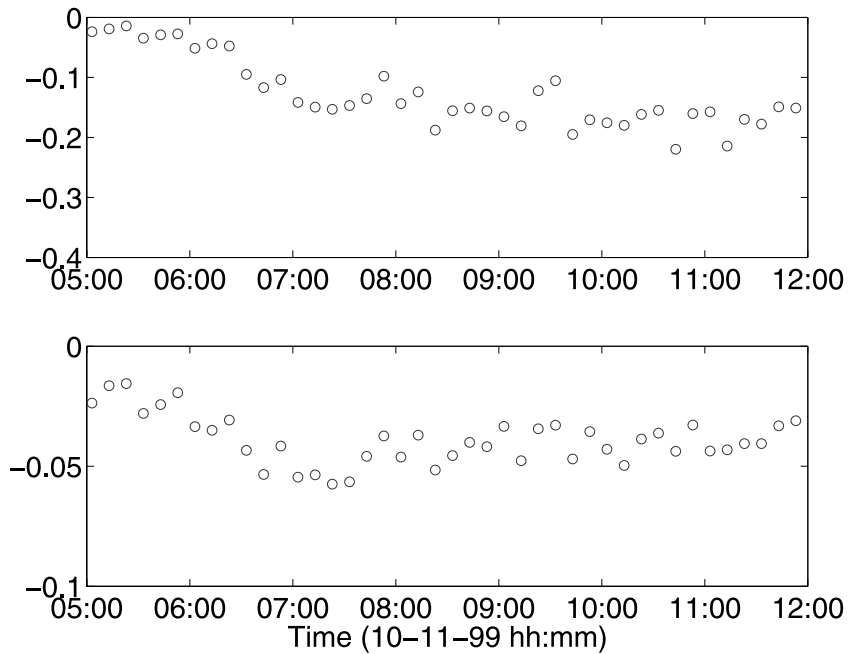

Figure 11. Temporal variation of the momentum flux $\overline{u^{\prime} w^{\prime}}$ (upper frame) and of the heat flux $\overline{w^{\prime} \theta^{\prime}}$ (lower frame) between 0500 and 1200 UTC during the low-level jet event. See color version of this figure in the HTML.

a new equilibrium had been established and the turbulence became homogeneous and stationary.

[41] A comparison for the stability functions $\phi_{m}$ and $\phi_{h}$ at a height of $3.25 \mathrm{~m}$ between the experimental values (circles) and the theoretical MOS results is shown in Figure 12. Predictions by the linear formulation (9) and (10) (squares) and by the general function (11) (stars) are in near-perfect agreement with the experimental data. The applicability of the MOS theory is undoubtedly due to the strong wind shear which continuously generates turbulence by mechanical production. The LLJ results in larger wind shear and this affects the turbulence below the jet center and near the ground surface. This large shear production of the turbulence at the wall totally overwhelms its buoyant destruction by the stable stratification. Thus with an ample supply of energy from the mean flow through the shear production, the turbulence can remain continuous and stationary, the two main requirements for the validity of the MOS theory.

[42] It is noted that MOS works well with strong wind shear in this LLJ event while it does not hold with strong wind shear in the K-H shear instability discussed in section 4.3. As explained, the strong wind shear is a prerequisite to feed energy to turbulence and makes turbulent flow continuously turbulent in the LLJ event. While the strong wind shear in the $\mathrm{K}-\mathrm{H}$ shear instability brings waves up along interface of stratified layers and makes these wave collapse. The turbulence, which in the beginning is relatively mild, is greatly intensified as a result of wave breaking. Strong wind shear plays different roles in these two events. It generates turbulence by mechanical production in the LLJ event and by wave breaking in $\mathrm{K}-\mathrm{H}$ shear instability. The flow is continuously turbulent in the LLJ event but the flow changes into turbulent in $\mathrm{K}-\mathrm{H}$ shear instability due to the strong shear.

\section{Concluding Remarks}

[43] Four atmospheric physical processes, namely a nonbreaking internal gravity wave, a K-H shear instability, a density current and a low-level jet, were identified and used to analyze the validity of the MOS theory in the SBL during CASES-99. The applicability of the MOS theory was determined by comparisons between the experimental gradient stability functions $\phi_{m}$ and $\phi_{h}$ and the theoretical MOS predictions with well-established flux profile relationships. Three categories of behavior can be observed:

[44] 1. Turbulence is intermittent for the duration of the entire event: the K-H shear instability.

[45] 2. Turbulence is intermittent in the development stage and stationary in the well-developed stage: the density current and the low-level jet.

[46] 3. Turbulence is not affected by the presence of the event: the nonbreaking internal gravity waves.

[47] The following conclusions can be drawn from this study:

[48] 1. The MOS theory can predict the surface fluxes properly during a nonbreaking internal wave event. No significant contribution to the background turbulence was observed from the wave turbulence interaction.

[49] 2. The surface fluxes cannot be correctly estimated by MOS during a K-H shear instability. Newly generated turbulence was found to occur during the wave breaking process.

[50] 3. The flux profile relationships hold well in the welldeveloped density current, but the head passage and the shear instability on the back of the density current will likely cause departures from MOS.

[51] 4. The MOS theory holds well in developed LLJ's in the present study. Their development stage will require more study.

[52] 5. The theoretical predictions give larger values of $\phi_{m}$ and $\phi_{h}$ in intermittent turbulence events involving shear instability; these lead to smaller surface fluxes and weaker turbulence transport than the experimental results.
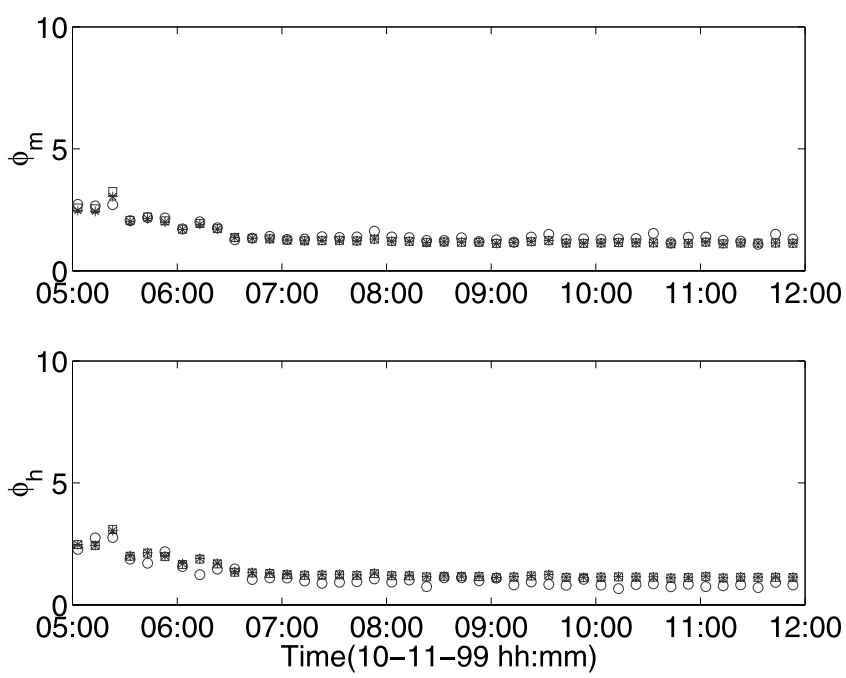

Figure 12. Comparisons of the experimental $\phi$ data points (circles) at $3.25 \mathrm{~m}$ above the ground with the theoretical predictions by the linear formulations (squares) and by the general stability function (stars) between 0500 and 1200 UTC during the low-level jet event. The structure of the figure is the same as that of Figure 3. See color version of this figure in the HTML. 
[53] On the basis of the comparisons of these four types of physical events in this study, MOS failure in the SBL appears to be primarily caused by K-H instability with strong but discontinuous and intermittent turbulence. However, new turbulence can also be generated during the head passage of a density current and the evolving stage of a LLJ. In general, MOS appears to be valid if the turbulence structure recovers after a 'disturbing event'. The conclusions drawn here may be of some use in the development of robust surface flux parameterizations for numerical models under stable conditions.

[54] The above conclusions are drawn based on 4 nonstationary events, namely, an internal gravity wave, a Kelvin-Helmholtz shear instability, a density current and a low-level jet. Further investigations will be necessary to verify these findings with a larger data set and with more precise flux estimation under nonstationary conditions.

[55] Acknowledgments. The authors gratefully acknowledge the use of the experimental data obtained by the National Center for Atmospheric Research (NCAR) and by the Environmental Technology Laboratory (ETL) of the National Oceanic and Atmospheric Administration (NOAA) during CASES-99. This research has been supported in part by the National Aeronautics and Space Administration (NASA-9426).

\section{References}

Banta, R. M., R. K. Newsom, J. K. Lundquist, Y. L. Pichugina, R. L. Coulter, and L. D. Mahrt (2002), Nocturnal low-level jet characteristics over Kansas during CASES-99, Boundary-Layer Meteorol., 105, 221 252

Benilov, A. Y., and B. N. Filyushkin (1970), Application of methods of linear filtration to an analysis of fluctuations in the surface layer of the sea, Izv. Russ. Acad. Sci. Atmos. Oceanic Phys., Engl. Transl., 6, 477482 .

Blumen, W., R. L. Grossman, and M. Piper (1999), Analysis of heat budget dissipation and frontogenesis in a shallow density current, BoundaryLayer Meteorol., 91, 281-306.

Blumen, W., R. M. Banta, S. P. Burns, D. C. Fritts, R. K. Newsom, G. S. Poulos, and J. Sun (2001), Turbulence statistics of a Kelvin-Helmholtz billow event observed in the nighttime boundary layer during the CASES-99 field program, Dyn. Atmos. Oceans, 34, 189-204.

Brutsaert, W. (1998), Land-surface water vapor and sensible heat flux: Spatial variability, homogeneity, and measurement scales, Water Resources Res., 34, 2433-2442.

Brutsaert, W. (1999), Aspects of bulk atmospheric boundary layer similarity under free-convective conditions, Rev. Geophys., 37, 439-451.

Brutsaert, W., and M. Sugita (1991), A bulk similarity approach in the atmospheric boundary layer using radiometric skin temperature to determine regional surface fluxes, Boundary-Layer Meteorol., 55, 1-23.

Cheng, Y., and W. Brutsaert (2005), Flux-profile relationships for wind speed and temperature in the stable atmospheric boundary layer, Boundary-Layer Meteorol., 114, 519-538.

Finnigan, J. J., F. Einaudi, and D. Fua (1984), The interaction between an internal gravity wave and turbulence in the stably-stratified nocturnal boundary layer, J. Atmos. Sci., 41, 2409-2436.

Gossard, E. E., and W. H. Hooke (1975), Waves in the Atmosphere: Atmospheric Infrasound and Gravity Waves-Their Generation and Propagation, 456 pp., Elsevier Sci., New York.
Hicks, B. B. (1976), Wind profile relations from the "Wangara" experiment, Q. J. R. Meteorol. Soc., 102, 535-551.

Hussain, A. K. M. F. (1983), Coherent structures-reality and myth, Phys. Fluids, 26, 2816-2849.

Hussain, A. K. M. F., and W. C. Reynolds (1972), The mechanics of an organized wave in turbulent shear flow, J. Fluid Mech., 160, 235256

Jiang, J., R. Street, and S. P. Klotz (1990), A study of wave-turbulence interaction by use of a nonlinear water wave decomposition technique, J. Geophys. Res., 95, 16,037-16,054.

Kondo, J., O. Kenechika, and N. Yasuda (1978), Heat and momentum transfer under strong stability in the atmospheric surface layer, J. Atmos. Sci., 35, 1012-1021.

Lapworth, A. (2002), Observations of atmospheric density currents using a tethered balloon-borne turbulence probe system, Boundary-Layer Meteorol., 101, 243-260.

Lenschow, D. H., X. S. Li, C. J. Zhu, and B. B. Stankov (1988), The stably stratified boundary layer over the Great Plains, Boundary-Layer Meteorol., 42, 95-121.

Lumley, J. L., and E. A. Terray (1983), Kinematics of turbulence convected by a random wave field, J. Phys. Oceanogr., 13, 2000-2007.

Mahrt, L., D. Vickers, R. Nakamura, M. R. Soler, J. Sun, S. Burns, and D. Lenschow (2002), Shallow drainage flows, Boundary-Layer Meteorol., $101,243-260$.

Newsom, R. K., and R. M. Banta (2002), Shear-flow instability in the stable nocturnal boundary layer as observed by Doppler lidar during CASES-99, J. Atmos. Sci., 60, 16-33.

Pahlow, M., M. B. Parlange, and F. Porte-Agel (2001), Monin-Obukhov similarity under stable atmospheric stability, Boundary-Layer Meteorol., 99, 225-248

Pope, S. B. (2000), Turbulent Flows, 806 pp., Cambridge Univ. Press, New York.

Poulos, G. S., et al. (2002), CASES-99: A comprehensive investigation of the stable nocturnal boundary layer, Bull. Am. Meteorol. Soc., 83, 555581 .

Simpson, J. E. (1997), Gravity Currents in the Environment and the Laboratory, 2nd ed., 244 pp., Cambridge Univ. Press, New York.

Smedman, A., M. Tjernstrom, and U. Högström (1993), Analysis of the turbulence structure of a marine low-level jet, Boundary-Layer Meteorol., $66,105-126$

Smedman, A., H. Bergstrom, and U. Högström (1995), Spectra, variances and length scales in marine stable boundary layer dominated by a low level jet, Boundary-Layer Meteorol., 76, 211-232.

Sugita, M., and W. Brutsaert (1992), The stability functions in the bulk similarity formulation for the unstable boundary layer, Boundary-Layer Meteorol., 61, 65-80.

Sun, J., et al. (2002), Intermittent turbulence associated with a density current passage in the stable boundary layer, Boundary-Layer Meteorol., $105,199-219$.

Webb, E. K. (1970), Profile relationships: The log-linear range, and extension to strong stability, Q. J. R. Meteorol. Soc., 96, 67-90.

Zhong, S., J. D. Fast, and X. Bian (1996), A case study of the Great Plains low-level jet using wind profiling network data and a high-resolution mesoscale model, Mon. Weather Rev, 124, 785-806.

W. Brutsaert and Y. Cheng, School of Civil and Environmental Engineering, Cornell University, Ithaca, NY 14853-3501, USA. (yc111@ cornell.edu)

M. B. Parlange, School of Architecture, Civil and Environmental Engineering, Ecole Polytechnique, Fédérale de Lausanne, Lausanne, CH1015, Switzerland. (Marc.parlange@epfl.ch) 